\title{
The validity of single item and domain specific sitting time questionnaires in a student population measured under free-living conditions
}

\author{
T.D. Atsma \\ Maastricht University \\ thomasatsma@hotmail.com
}

\begin{abstract}
Sedentary time questionnaires provide a low cost, low participant burden way of assessing sedentary behavior, but their subjectivity is questionable. The aim of this study was to assess the validity of sedentary time questionnaires, both single item and domain specific, in a student population under free living conditions with accelerometry as criterion measure. It is hypothesised that both sedentary time questionnaires underestimate the sitting time. Twenty healthy subjects ( 15 male, 5 female) participated in a one-week observational study under free-living conditions. They wore an accelerometer (activPAL) continuously for 7 consecutive days and afterwards filled in a single item (IPAO) and a domain specific sedentary time questionnaire (dsSTO) about the same time period. Spearman correlations were used to assess relative validity and Wilcoxon signed-rank tests and Bland-Altman plots were used to assess absolute agreement between questionnaires and accelerometry. Sitting time was significantly underestimated by the IPAO and insignificantly overestimated by the dsSTO compared to accelerometry for the full week average sitting times. Correlations between questionnaires and accelerometry were weak to moderate. For both questionnaires there were large individual differences in estimations compared to accelerometry. The correlation between questionnaire and accelerometer determined sitting events lasting 30 minutes or longer was moderate. The dsSTQ is the more accurate measure of the two questionnaires on sitting time on the population level. However, there were large individual differences in estimations, and thus it does not seem to be a valid, reliable measurement tool for sitting time in small populations and in studies were sitting time is an important outcome. For these studies, accelerometry or more preferably direct observation provides a better estimate of sitting time.
\end{abstract}




\section{Keywords}

Sedentary time questionnaires, accelerometry, activPAL, validation study, IPAQ, single item, domain specific, students

\section{Introduction}

In the last century, new technological innovations and changed living and working environments have led to an increasingly sedentary lifestyle in the developed countries ( 1 , 2). The on going trend of increasing obesity and diabetes prevalence is a concern for global health and the economy (1-4). In order to change this trend, changes in lifestyle need to be made. To implement these changes in the best possible way, it is useful to first investigate the current lifestyle profiles of specific populations. To get an insight in sedentary behavior on the population level, different kinds of monitoring tools exist, such as questionnaires and accelerometry. It is of importance to assess the validity of these monitoring tools. To assess sedentary behavior self-report measures like questionnaires, surveys and diaries are often used because of their low cost, practicality and low participant burden (5). The downside of self-report measures is the issue of recall and response bias which could lead to an over- or underestimation of the sedentary behavior (6). Direct measures, such as calorimetry, accelerometer, heart rate measurements and direct observation, are thought to be more precise measures of sedentary behavior as there is no risk for recall or response bias. The major downside of direct measures compared to self-report measures are the higher costs which makes them difficult to apply to large population studies (5). The question arises how accurate sedentary behavior questionnaires are. To get an answer to this question, studies which compare the sedentary time outcomes of self-reported and accelerometer measures are needed. Since age and education level are probable to have an effect on the level and pattern of sedentary behavior, the studies should be performed in a variety of population samples. One example of a specific population is the student population, which is often classified by high education levels and irregular lifestyles without regular working schedules. Work makes physical activity and sedentary time easier to report, because of a more constant schedule during working days. Students often lack the 9 till 5 structure of work and educational programs often have irregular hours, which provide the students with irregular spare time. Because self-studying is performed on a free basis, there is possibly a larger spread of physical activity. This irregularity raises the question whether sedentary time questionnaires provide a valid outcome in a student population. Therefore, the primary aim of this study was to assess the validity of sedentary time questionnaires, both single item and domain specific, in a student population with accelerometry as criterion measure. It is hypothesised that the single item (IPAO) and 
the domain specific sedentary time questionnaires (dsSTO) both underestimate the sedentary time compared to accelerometry and that there is little agreement between the questionnaires and accelerometry.

\section{Material and Methods}

\section{Subjects}

Twenty healthy students participated in this study. Participants had to be students, with a BMI between 18 and 25, and at least 18 years of age. Exclusion criteria were movement disabilities and diseases interfering with physical activity.

\section{Study design}

This study was an observational study comparing accelerometer and questionnaire determined sitting time for seven consecutive days under free-living conditions. The subjects wore an accelerometer (activPAL3 ${ }^{\text {TM }}$, PAL Technologies, Glasgow, Scotland) on the thigh and filled in a questionnaire about the last seven days right before attaching the accelerometer, directly after the removal and as a retest seven days after the removal of the accelerometer. Two questionnaires were used in the study, of which the first questionnaire was the Dutch translation of the International Physical Activity Questionnaire (IPAO) in the Long Form about the last seven days, of which only the last question on the average sitting time on weekdays and on weekend days in the last 7 days was used for further analysis. In addition to the IPAO, a domain specific sedentary time questionnaire (dsSTQ) was used. The dsSTO split sitting time into sitting while travelling, working, watching TV, using the computer in the spare time, during activities in the spare time, and eating. It was asked to fill in the average daily sitting time for the last 7 days and the questions were asked for weekdays and weekend days separately.

\section{Procedure}

Two meetings with the subjects took place. At the first meeting, the subjects filled in the questionnaire. Length, weight and age of the subjects were asked. After the questionnaire was completed, the accelerometer was attached to the right thigh. The subjects were able to sleep, shower and bathe with the accelerometer and were instructed to remove the monitor only when it was necessary because of skin irritations or water- or other activities with a high risk of losing the monitor. The subjects were informed on how to reattach the accelerometer themselves in case it got loose and they were provided with additional film to reattach the accelerometer. It was emphasised to the subjects to maintain normal 
activities during the upcoming week. The subjects kept a log of their sleeping times for the seven measuring days with an accuracy of 10 minutes, which were used as guidance for obtaining the sleeping times from the accelerometer data. Sleeping times were logged in order to calculate the sitting/lying time while awake by subtracting the sleeping time from the total sitting time, which was obtained with the accelerometer. Seven days after the first meeting the second meeting took place. The accelerometer was removed and afterwards the subjects were asked to fill in the online questionnaire again. The subjects were reminded that within seven days they would receive an email with the re-test questionnaire. In this email it was emphasised that the questionnaire had to be filled in immediately on the day of receiving the email.

\section{Data analysis}

Subjects with less than four consecutive weekdays or less than two weekend days of complete data were excluded from further analysis. The sitting times per day calculated by the activPAL analysis software (v7.2.29, Research Edition) were collected into one Excel worksheet to calculate average weekday, weekend day and total week average sitting times. The sleeping times were subtracted from the total sitting/lying time collected by the activPAL in order to obtain the waking sitting times. The accelerometer data of the first and the eighth measuring day were summed in order to create seven complete days of data. The questionnaire data of the second meeting was compared to the accelerometer data because they covered the same week. The activPAL was used as the criterion measure for the validation of the questionnaires. The average sitting time outcomes of the IPAO and the dsSTO were compared with the accelerometer outcomes for weekdays, weekend days and total average of the week. The dsSTO and accelerometer determined average number of sitting events lasting longer than 30 minutes were compared for weekdays, weekend days and total week average. Spearman's correlation coefficient were calculated to assess the association, Wilcoxon signed-rank tests were used to assess the significance of the differences between the methods and Bland-Altman plots were created to assess the absolute agreement between the methods. In the Bland-Altman plots, a difference above zero indicated an underestimation and a difference below zero indicated an overestimation of the questionnaire compared to accelerometry determined sitting. Lower bias and smaller $95 \%$ limits of agreement indicated better agreement. When the plot seemed to display a proportional bias, mean difference and limits of agreement were also assessed with regression (7). The statistical analyses were conducted with IBM SPSS Statistics 22 (SPSS, Inc., Chicago, IL) and a p<0.05 was set as statistically significant. The sitting events lasting longer than 30 minutes without at least 2 minutes of breaking were 
determined manually with the activPAL software output per day. When a break longer than 2 minutes occurred in the middle of a longer sitting period, it was measured whether the sitting time before and after the break was minimally 30 minutes. In such a case they were counted as two separate sitting events lasting longer than 30 minutes. When the break was shorter than 2 minutes, the sitting time before and after the break were counted as one event. When more breaks shorter than 2 minutes occurred in a sitting period, the break times were summed. When the sum of the break times reached 2 minutes, the sitting event stopped at the last break of the sum, and the sitting time after the last break was counted as a new sitting event. When the sitting events were less than 30 minutes, they were not counted. Sleeping was not counted as a sitting event.

\section{Results}

\section{Subject characteristics}

A total of twenty subjects ( 15 male, 5 female) participated in this study, with a mean age of $21 \pm 2$ years, an average height of $1.78 \pm 0.09 \mathrm{~m}$ and an average weight of $68 \pm 8 \mathrm{~kg}$, which resulted in an average $\mathrm{BMI}$ of $21.3 \pm 1.2$.

\section{Population average sitting times for the three measuring methods}

For full week averages, the population average sitting times and the range of individual values derived from the three measuring methods were 575.7 (450.5 - 680.6), 383.4 (102.9 - 677.1), and 572.5 (263.6 - 840.7) min/day for accelerometry, IPAO and dsSTO respectively (table 1). Both IPAQ and dsSTO derived sitting times had broader ranges of individual values than accelerometry determined sitting times. On the population average, sitting times on weekdays were higher than sitting times in the weekend for all three measuring methods.

Table 1: Population average sitting times of the three measuring methods

\begin{tabular}{|c|c|c|c|c|c|c|}
\hline & \multicolumn{2}{|c|}{ Accelerometry (activPAL) } & \multicolumn{2}{|l|}{ IPAQ } & \multicolumn{2}{|l|}{ dsSTQ } \\
\hline & $\begin{array}{l}\text { Sitting time } \\
\text { (min/day) }\end{array}$ & $\begin{array}{l}\text { Range } \\
\text { (min/day) }\end{array}$ & $\begin{array}{l}\text { Sitting time } \\
\text { (min/day) }\end{array}$ & $\begin{array}{l}\text { Range } \\
\text { (min/day) }\end{array}$ & $\begin{array}{l}\text { Sitting time } \\
\text { (min/day) }\end{array}$ & $\begin{array}{l}\text { Range } \\
\text { (min/day) }\end{array}$ \\
\hline Full week & 575.7 & $450.5-680.6$ & 383.4 & $102.9-677.1$ & 572.5 & $263.6-840.7$ \\
\hline Weekdays & 587.8 & $411,4-707,3$ & 403,5 & $120-660$ & 610 & $285-1050$ \\
\hline Weekend & 522.8 & $373,0-682.5$ & 333 & $60-720$ & 478.8 & $180-780$ \\
\hline
\end{tabular}

\section{ActivPAL and IPAQ sitting time correlation and agreement}

There was moderate correlation between accelerometer and IPAO determined sitting time for the full week averages $(\rho=0.442, P=0.114)$, and weak correlation for weekday averages 
$(\rho=0.346, P=0.174)$ and weekend averages $(\rho=0.305, P=0.251)$. Positive biases for full week, weekday and weekend averages (175.3, 174.8 and 189.1 sitting min/day respectively) were found which were significantly different from zero (figure 1). The $95 \%$ limits of agreement for full week sitting time averages were -107.4 and $458.0 \mathrm{~min} /$ day as is depicted in figure 1 . A linear regression $(r=0.764)$ was performed and $95 \%$ limits of agreement are represented with the equation: Difference $=723-1.12 *$ Mean $\pm 1.96 * 97.0\left(\mathrm{SD}_{\mathrm{r}}\right) \mathrm{min} /$ day.

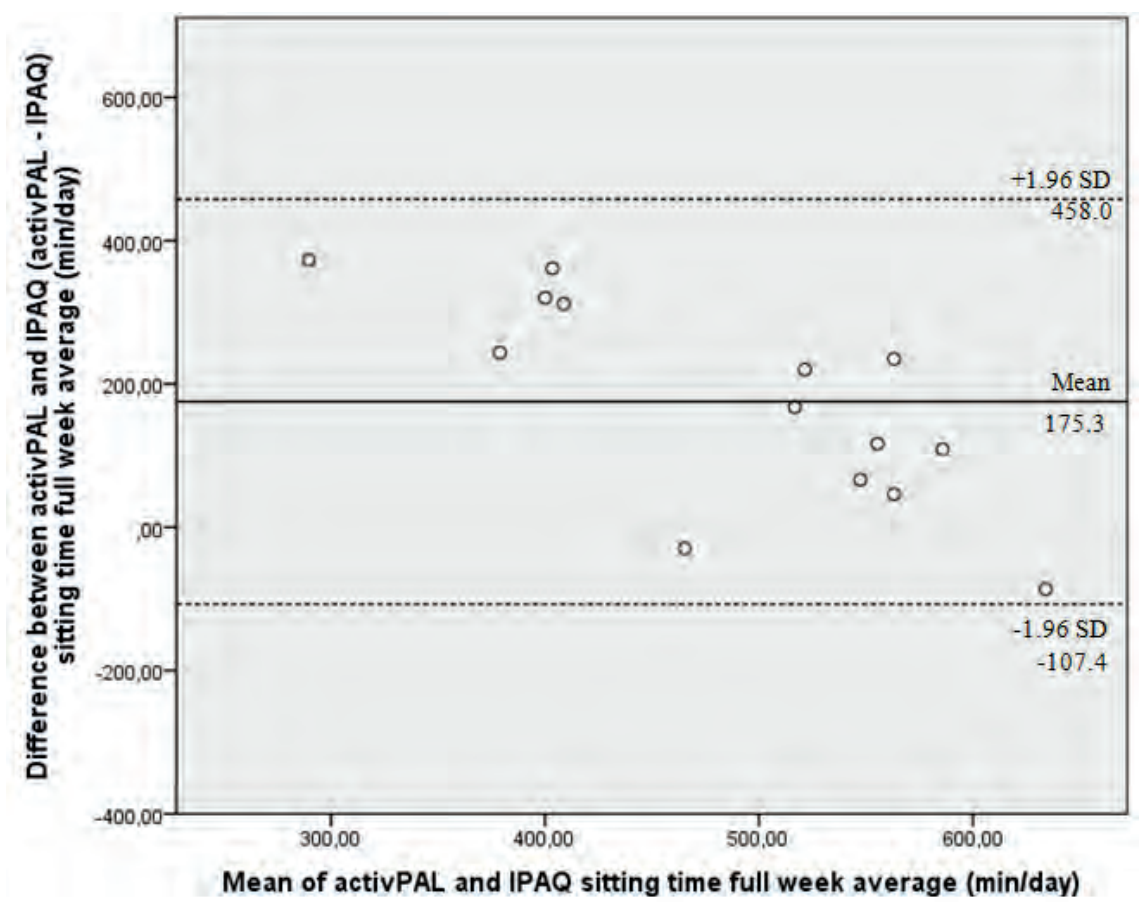

Figure 1. Bland-Altman plot of the full week average sitting times measured with the activPAL and the IPAO (IPAQ sitting time was subtracted from activPAL sitting time). Dashed lines are the $95 \%$ limits of agreement and the black line is the mean difference between the methods. The grey dashed line indicates zero difference between the methods.

\section{ActivPAL and dsSTO sitting time correlation and agreement}

Accelerometer and dsSTO determined sitting had moderate correlation for the full week averages $(\rho=0.482, P=0.081$ ), weak correlation for weekday averages $(\rho=0.366, P=0.149)$ and weekend averages $(\rho=0.368, P=0.161)$. Mean differences between accelerometer and dsSTQ were insignificantly different from zero with values of $-22.9,-53.1$ and 46.9 sitting 
$\mathrm{min} /$ day for full week, weekday and weekend averages respectively (figure 2). The $95 \%$ limits of agreement for the full week average sitting time were -290.7 and $244.9 \mathrm{~min} /$ day (figure 2). This is less bias than the bias of IPAO determined sitting time compared to accelerometry. Both higher and lower estimates of sitting time have been observed for the dsSTO compared to accelerometry, as is visible in figure 2. There seemed to be a proportional bias in the plots, and thus a linear regression $(r=0.764)$ was performed and $95 \%$ limits of agreement are represented with the equation: $610-1.08$ * Mean \pm 1.96 * $99.19 \mathrm{~min} /$ day.

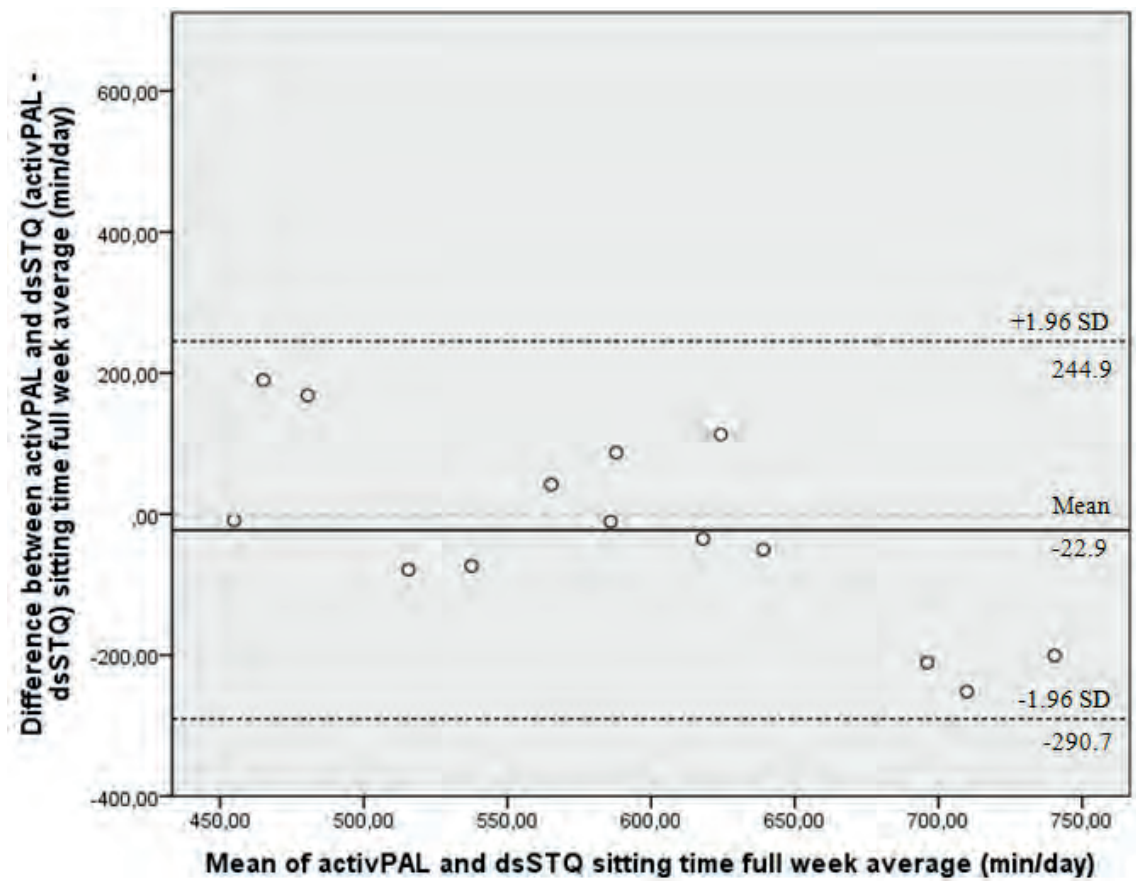

Figure 2. Bland-Altman plot of the full week average sitting times measured with the activPAL and the dsSTO (dsSTO sitting time was subtracted from activPAL sitting time). Dashed lines are the $95 \%$ limits of agreement and the black line is the mean difference between the methods. The grey dashed line indicates zero difference between the methods.

IPAO and dsSTO sitting time in relation to the activPAL sitting time

When figure 1 and 2 are merged and the $y$-axis is translated to the $x$-axis, it is more visible that the IPAO on average underestimates sitting time compared to accelerometry and that 
the dsSTQ insignificantly overestimates sitting time compared to accelerometry (figure 3).

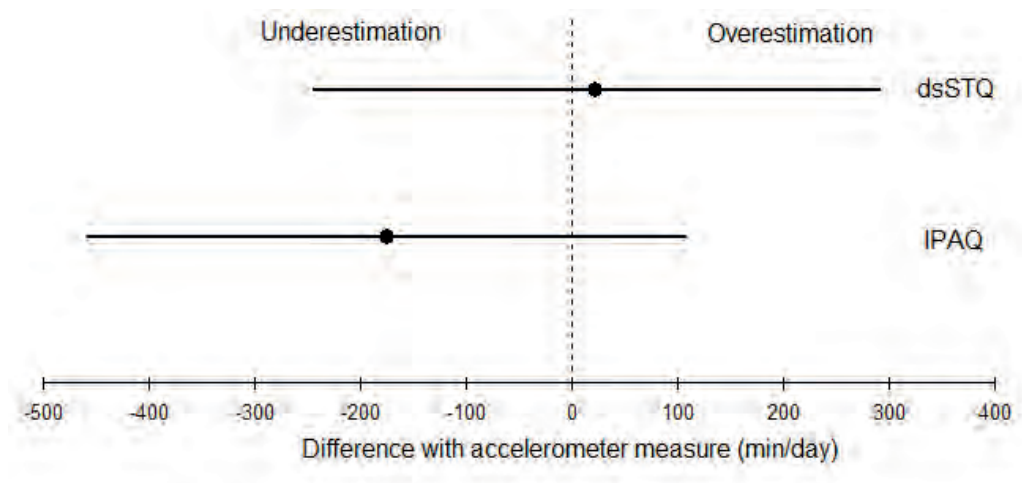

Figure 3. Plot of the differences between questionnaire (dsSTO and IPAO) and accelerometer full week average sitting times (questionnaire - accelerometer). Dots represent the mean differences and bars the $95 \%$ limits of agreement.

ActivPAL and dsSTQ sitting events lasting 30 minutes or longer correlation and agreement The correlation between accelerometer and dsSTO determined sitting events lasting 30 minutes or longer was weak for full week averages $(\rho=0.399, P=0.176)$, moderate for weekday averages $(\rho=0.450, P=0.080)$, and weak correlation for weekend averages $(\rho=0.387, P=0.154)$. Negative biases for full week, weekday, and weekend averages of sitting events lasting 30 minutes sitting or longer have been found, with mean differences of -2.2, -2.4 and -1.5 events respectively which were significantly different from zero (figure 4). Data of one subject was omitted from analysis due to a misinterpretation of the question, which resulted in questionnaire values which were double the average of the population. 


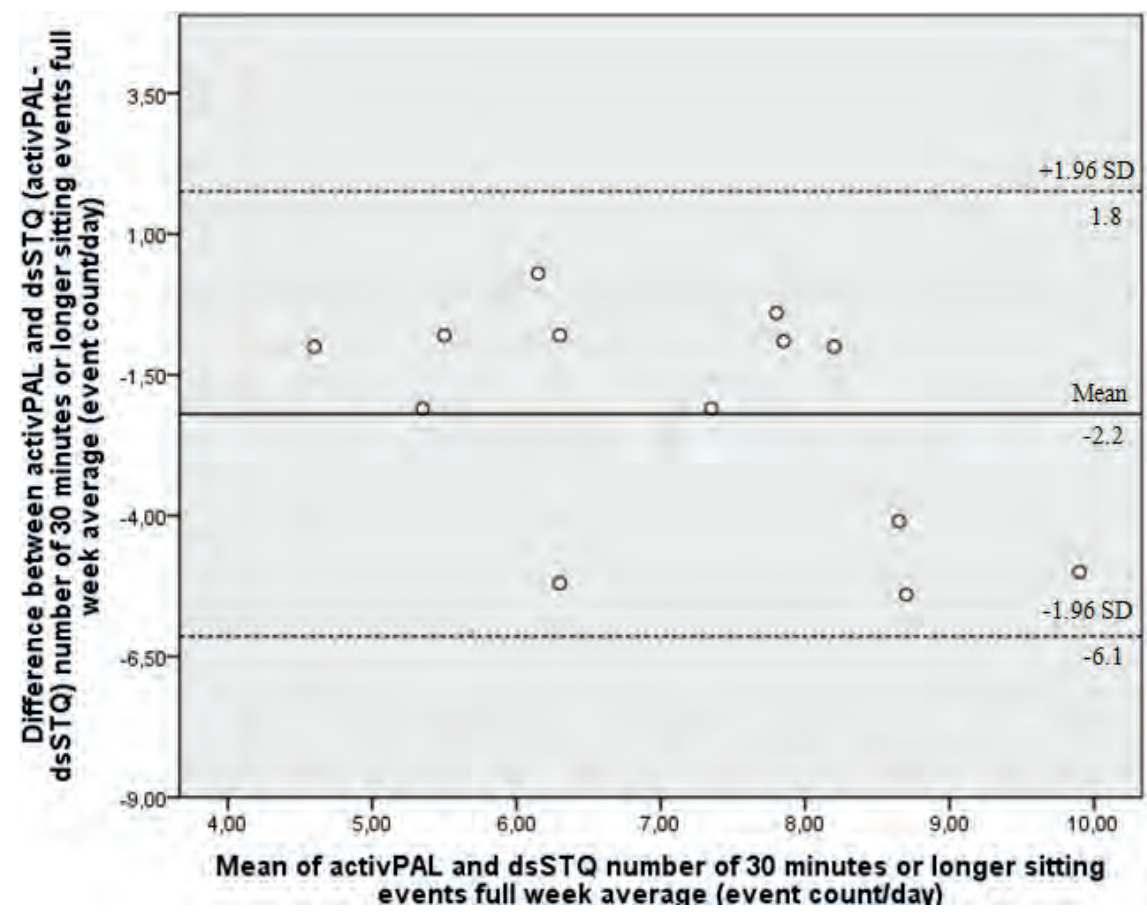

Figure 4. Bland-Altman plot of full week average sitting events lasting 30 minutes or longer measured with the activPAL and the dsSTO (dsSTO sitting events were subtracted from activPAL sitting events). Dashed lines are the $95 \%$ limits of agreement and the black line is the mean difference (-2.2 events) between the methods. The grey dashed line indicates zero difference between the methods.

\section{Discussion/Conclusion}

The aim of this study was to assess the validity of the IPAQ and the dsSTQ outcomes in relation to the activPAL accelerometer sitting time outcomes. It was hypothesised that the IPAO and the dsSTO underestimated sitting time compared to accelerometry determined sitting time and that there would be little agreement. The major findings of this study were that the IPAO significantly underestimated and the dsSTO insignificantly overestimated sitting time for the full week averages compared to accelerometry and that the correlations between the methods were weak to moderate.

In the current study, sitting time was significantly underestimated by the IPAO compared to accelerometry. This is in accordance with previous studies (8-10). In a study by KozeyKeadle et al. (9) there was a significant underestimation of sitting time with the IPAO for weekend days, but an insignificant underestimation for weekdays compared to activPAL 
accelerometry. However, in a comparable study by Clemes et al.the differencewas significant for weekdays, which is in accordance with the findings of the current study. In the current study, the dsSTO insignificantly overestimated sitting time compared to accelerometry for full week average and weekdays, but there was an insignificant underestimation of sitting time on weekend days. The overestimation of sitting time on weekdays by domain specific questionnaires compared to accelerometry is also found in previous studies (8, $9,11)$. However, in a study by Marshall et al. (12) there was a significant underestimation of sitting time on weekdays instead of an insignificant overestimation, with a mean difference of $63.6 \mathrm{~min} /$ day between questionnaire and accelerometer measurements. A possible explanation for the different findings could be the difference in questionnaires. The current study used the same questionnaire as used by Marshall et al., but with an added question on time spent sitting while eating. The addition of the question most likely resulted in higher total sitting time reports, because in the current study subjects reported considerable amounts of time spent sitting while eating (on average $41 \mathrm{~min} /$ day). It could be that the underestimation of sitting time on weekdays found by Marshall et al. would be insignificant or absent if they had added the question on sitting while eating. A comparable explanation can be used for the different findings of the study by Scholes et al. (13), which also found a significant underestimation of sitting time. The study by Scholes et al. used a limited questionnaire with only questions regarding sitting while watching television, sitting during non-television leisure time, and sitting while working, which do not cover all possible sitting situations. The correlations between the dsSTQ and accelerometry were insignificant, which is in accordance with some studies $(9,12)$, while Wijndaele et al. (11) found a significant correlation between domain specific sitting time questionnaires and accelerometry $(\rho=0.52, P<0.001)$ for full week sitting averages. One possible explanation for the higher correlation found in Wijndaele's study compared to the correlation in the current study is the use of an even more extensive domain specific sitting time questionnaire, which also included separate questions on sitting during leisure time activities, such as sitting while reading, socialising, and listening to music.

Another outcome of this study was the number of sitting events lasting 30 minutes or longer. It was found that the dsSTO significantly overestimated the number of sitting events compared to accelerometry for both weekdays (2.4 events/day) and weekends ( 1.5 events/day) and the correlations between the methods were weak to moderate. To the author's knowledge, the validity of such a questionnaire outcome has not been studied before. However, there have been studies on breaks in sedentary time, which is a related outcome, considering that the number of sitting events lasting 30 minutes or longer depends on the number and timing of breaks. From the results of the current study, it can 
be concluded that the used questionnaire is not a valid measurement tool for the number of sitting events lasting 30 minutes or longer compared to accelerometry. It is however unknown how accurate the used criterion measure is in measuring the length of sitting events. Some studies have validated accelerometry determined number of breaks with direct observation as criterion measure $(14,15)$. They found that the activPAL estimated the absolute number of breaks and the number of sitting to standing transitions precisely and accurately. However, the way the activPAL data is presented with the software gave some difficulties in the current study with determining sitting and breaking time. It was not possible to obtain the exact length of the small breaks occurring in sitting events because sitting, standing and walking times were only provided for epochs of 1 or 24 hours. Therefore the assessment of the total length of breaks in the sitting events with the activPAL was difficult and the manual determination could have led to incorrect classifications of sitting events as matching the criteria of 30 minutes or longer without at least two minutes of breaks. Future studies combining direct observation and accelerometry should be performed to validate the outcome measure of sitting events with different criteria for sitting duration and breaking time.

It is important to note that by stating questionnaire outcomes over- or underestimate sitting time, it is implied that accelerometry provides the 'gold standard'. However, accelerometers have their biases and errors in measuring sitting time as well (16). In a validation study by Kozey-Keadle et al. (17) it was found that both activPAL and ActiGraph (cut point <100 counts per minute), provided an underestimation of sitting time compared to direct observation. However, the underestimation was larger with the ActiGraph than with the activPAL, with differences of $4.9 \%$ and $2.8 \%$ respectively compared to direct observation (17). In a later study by Kozey-Keadle et al. (9) in which the ActiGraph outcomes were compared to the activPAL outcomes, it was found that the ActiGraph underestimated sitting time on weekdays compared to the activPAL outcomes but it overestimated sitting time on weekend days. This difference between weekdays and weekend days could be explained by the observed higher time spent standing during the weekend together with the inability of the ActiGraph to distinguish between standing and sitting (9). Comparisons between the findings of the current study with studies, which used the ActiGraph, should thus be made with caution. Considering the difference found between direct observation and accelerometry determined sitting time, the underestimations of sitting time by questionnaires found in the current study could in reality be larger and overestimations could be equally smaller, since the 'goldstandard' of this study already underestimated sitting time. Future studies should combine direct observation, accelerometry and questionnaires for the best validation of the sitting time questionnaires. 
One of the major strengths of the current study was the continuous wearing of the accelerometers instead of shorter wear times, which have been used in most other studies. Another strength of the current study is the measurement of sedentary behavior under free-living conditions. Subjects were free to do what they wanted and did not have to write down what kind of activities they did during the week. Furthermore, the use of the activPAL instead of the often-used ActiGraph is the strength of the current study, since it is able to distinguish between standing still and sitting, which is important in measuring sedentary time. However, there were some limitations of the current study. The domain specific questionnaire used in the current study split the questions into sitting times on working days and free days. For the data analysis, it was assumed that working days were equal to weekdays, and free days were equal to weekend days, but this is not always true for students. Students often have part time jobs in addition to their education, which can also occur in the weekends. Therefore, the distinction between working days and free days could be effective for working adults, but for a student population, a distinction between weekdays and weekend would be more suitable. Future studies in student populations should thus assess the validity domain specific questionnaires with the distinction between weekdays and weekend days or the subjects need to be asked which days of the week they considered as working days and which as free days. Furthermore, the subjects in the current study were a convenience sample and were not selected to represent the student population as a whole. The majority of the subjects studied health sciences, and thus could possibly have more knowledge on the average amount spent sitting which would reflect on better estimates with the single item question compared to the estimates of more heterogeneously educated population samples. Therefore, future studies should include more heterogeneous student populations for a better validation of sitting time questionnaires in the average student population.

In conclusion, it was found that dsSTQ insignificantly overestimated sitting time while the IPAO significantly underestimated sitting time compared to accelerometry, which makes the dsSTQ the more accurate tool of the two for measuring sitting time on the population level. However, there were large individual differences in estimations, and thus it does not seem to be a valid, reliable measurement tool for sitting time in small populations and in studies were sitting time is an important outcome. For these studies, accelerometry or more favorably direct observation is advised. The number of sitting events lasting 30 minutes was overestimated by the questionnaire compared to accelerometry, but accelerometry determination of sitting events was difficult. Therefore, future studies combining direct observation and accelerometry should be performed to validate the outcome measure of sitting events. 


\section{Role of the student}

Thomas Atsma was an undergraduate student in Biomedical Science who performed a research project together with undergraduate student Tom Timmermans under supervision of Tom Melai of the Human Movement Sciences Department of Maastricht University. The topic was proposed by Hans Savelberg. The complete research was done by Atsma and Timmermans with an equal spread of practical work including designing the questionnaires, working with the subjects and processing the results. Writing was done separately with a different focus as distinction: Atsma focussed on inactivity while Timmermans focussed on activity.

\section{Acknowlegdements}

I want to thank Hans Savelberg for lending the activPALs to make this research possible. I also want to thank Tom Melai and Bernard Duvivier for their helpful comments and guidance during this research. And last but not least, I also like to thank all the participants who were willing to wear the cumbersome device through all kinds of weather conditions. Without them, this research would not have been possible.

\section{References}

1. Kohl 3rd HW, Craig CL, Lambert EV, Inoue S, Alkandari JR, Leetongin G, et al. The pandemic of physical inactivity: global action for public health. The Lancet. //;380(9838):294-305.

2. Caballero B. The global epidemic of obesity: an overview. Epidemiologic reviews. 2007;29:1-5. PubMed PMID: 17569676 .

3. Wild S, Roglic G, Green A, Sicree R, King H. Global prevalence of diabetes: estimates for the year 2000 and projections for 2030. Diabetes care. 2004;27(5):1047-53.

4. Katzmarzyk PT, Gledhill N, Shephard RJ. The economic burden of physical inactivity in Canada. CMAJ : Canadian Medical Association journal = journal de l'Association medicale canadienne. 2000 Nov 28;163(11):1435-40. PubMed PMID: 11192648. Pubmed Central PMCID: 80410.

5. Prince SA, Adamo KB, Hamel ME, Hardt J, Connor Gorber S, Tremblay M. A comparison of direct versus selfreport measures for assessing physical activity in adults: a systematic review. The international journal of behavioral nutrition and physical activity. 2008;5:56. PubMed PMID: 18990237. Pubmed Central PMCID: 2588639 .

6. Shephard RJ. Limits to the measurement of habitual physical activity by questionnaires. British journal of sports medicine. 2003 Jun;37(3):197-206; discussion PubMed PMID: 12782543. Pubmed Central PMCID: 1724653

7. Bland JM, Altman DG. Measuring agreement in method comparison studies. Statistical methods in medical research. 1999 Jun;8(2):135-60. PubMed PMID: 10501650.

8. Clemes SA, David BM, Zhao Y, Han X, Brown W. Validity of two self-report measures of sitting time. Journal of physical activity \& health. 2012 May;9(4):533-9. PubMed PMID: 21946087.

9. Kozey-Keadle S, Libertine A, Staudenmayer J, Freedson P. The Feasibility of Reducing and Measuring Sedentary Time among Overweight, Non-Exercising Office Workers. Journal of obesity. 2012;2012:282303. PubMed PMID: 22175004. Pubmed Central PMCID: 3228288. 
10. Dyrstad SM, Hansen BH, Holme IM, Anderssen SA. Comparison of self-reported versus accelerometermeasured physical activity. Med Sci Sports Exerc. 2014 Jan;46(1):99-106. PubMed PMID: 23793232.

11. Wijndaele K, De Bourdeaudhuij I, Godino JG, Lynch BM, Griffin SJ, Westgate K, et al. Reliability and Validity of a Domain-Specific Last-7-Day Sedentary Time Questionnaire. Med Sci Sports Exerc. 2014 Jan 30. PubMed PMID: 24492633 .

12. Marshall AL, Miller YD, Burton NW, Brown WJ. Measuring total and domain-specific sitting: a study of reliability and validity. Med Sci Sports Exerc. 2010 Jun;42(6):1094-102. PubMed PMID: 19997030.

13. Scholes S, Coombs N, Pedisic Z, Mindell JS, Bauman A, Rowlands AV, et al. Age- and sex-specific criterion validity of the health survey for England physical activity and sedentary behavior assessment questionnaire as compared with accelerometry. American journal of epidemiology. 2014 Jun 15;179(12):1493-502. PubMed PMID: 24863551. Pubmed Central PMCID: 4051878.

14. Lyden K, Kozey-Keadle S, Staudenmayer J, Freedson P. Validity of two wearable monitors to estimate breaks from sedentary time. Med Sci Sports Exerc. 2012;44(11):2243-52

15. Grant PM, Ryan CG, Tigbe WW, Granat MH. The validation of a novel activity monitor in the measurement of posture and motion during everyday activities. British journal of sports medicine. 2006 Dec;40(12):992-7. PubMed PMID: 16980531. Pubmed Central PMCID: 2577473.

16. Healy GN, Clark BK, Winkler EA, Gardiner PA, Brown WJ, Matthews CE. Measurement of adults' sedentary time in population-based studies. Am J Prev Med. 2011 Aug;41(2):216-27. PubMed PMID: 21767730. Pubmed Central PMCID: 3179387 .

17. Kozey-Keadle S, Libertine A, Lyden K, Staudenmayer J, Freedson PS. Validation of wearable monitors for assessing sedentary behavior. Med Sci Sports Exerc. 2011 Aug;43(8):1561-7. PubMed PMID: 21233777. 